\section{Prevalência de cárie dentária em escolares de 12 e 15 anos de Salvador, Bahia, 2001}

\author{
Dental caries prevalence of schoolchildren \\ of 12 and 15 years old in Salvador, Bahia, \\ 2001
}

Maria Cristina Teixeira Cangussu 1 Fernandez Roberto Augusto Castellanos 2

1 Faculdade de Odontologia. Universidade Federal da Bahia. Rua Araújo Pinho, 62. Canela. Salvador, BA, Brasil. CEP: 41.110-150 E-mail: cangussu@ufba.br

2 Faculdade de Saúde Pública. Universidade de São Paulo. São Paulo, SP, Brasil

\begin{abstract}
Objectives: to determine dental caries prevalence and severity of schoolchildren 12 and 15 years old in Salvador, in different health districts.

Methods: a transversal study with multiple stage raffled probabilistic sample, pre-stratified by the 12 health districts. With a team of previously calibrated examiners, 3313 students were examined (DMFT). Data on access and coverage of healthcare services were obtained from a questionnaire. In the analysis we used: Chi-square test, Student t test, bivariate analysis, and non-conditional logistic regression, using a significance level of $5 \%$ for all statistical testing.

Results: $49.6 \%$ of the sample had had access to dental care during the previous year. Almost $40 \%$ of 12 year old students and $27.7 \%$ of the 15 year old students reported participation in collective programs in the last two years. A DMFT of 1.44 was determined and $49 \%$ of 12 year old caries-free children and an index of 2.66 for the 15 year old students. The districts with lower caries prevalence were Pau da Lima, Cajazeiras and Subúrbio Ferroviário.

Conclusions: the prevalence of dental caries is low among schoolchildren in different districts, although variations have been determined in the dental caries distribution of different geographical areas.
\end{abstract}

Key words Dental caries, DMF index, Health surveys, Adolescent

\section{Resumo}

Objetivos: determinar a prevalência e severidade da cárie em escolares aos 12 e 15 anos de idade em Salvador, segundo distrito sanitário.

Métodos: desenvolveu-se um estudo transversal com uma amostra probabilística, pré-estratificada pelos 12 distritos. Equipes calibradas examinaram 3313 escolares (CPO-D). Dados sobre acesso e cobertura dos serviços de saúde foram obtidos através de questionário. Para a análise utilizaram-se: teste do quiquadrado, $t$ de Student, análise bivariada e de regressão logística com nível de significância de 5\%.

Resultados: 49,6\% da amostra teve acesso ao dentista no último ano e quase $40 \%$ aos 12 anos e $27,7 \%$ aos 15 anos relataram ter participado de programas coletivos nos últimos dois anos. Observou-se um CPO-D de 1,44 e 49\% das crianças livres de cárie aos 12 anos, e um índice de 2,66 aos 15 anos. Os distritos com menor prevalência de cárie foram o de Pau da Lima, Cajazeiras e Subúrbio Ferroviário.

Conclusões: foi baixa a prevalência de cárie, $e$ são encontradas iniqüidades na distribuição da cárie em áreas geográficas distintas, reforçando a necessidade de vigilância à saúde.

Palavras-chave Cárie dentária, Índice CPO, Levantamentos epidemiológicos, Adolescente 


\section{Introdução}

O objetivo final da epidemiologia, independente dos métodos utilizados, é contribuir para a melhoria da saúde das populações, tornando possível, a partir da produção e acúmulo de dados empíricos e conhecimentos, direcionar a tomada de decisão em saúde pública. Uma das possibilidades de estudo é o uso do espaço geográfico como categoria analítica, que permitiria compreender, de forma dinâmica, a complexa relação e interações que permeiam a ocorrência da saúde e doença na população. ${ }^{1}$ Barmes 2 afirma que, de modo geral, os dados e a lógica com que são coletadas as informações na saúde bucal não tem privilegiado o planejamento, programação e definição de estratégias para o controle das doenças e melhoria do estado de saúde das populações.

A cárie é uma das principais patologias de interesse em odontologia e além dos fatores físicos e biológicos da cavidade bucal que contribuem para sua determinação, têm fatores comportamentais, demográficos e sócio-culturais como hábitos de higiene e dietéticos, idade, sexo, e o grau de desenvolvimento do país e seus investimentos nos setores saúde e educação como intervenientes. 3

A partir da década de 90, os apontam na detecção de piores condições de saúde bucal em crianças de menor renda, sendo que já se identificou que o acesso a fluoretos, através da fluoretação das águas de abastecimento público ou da pasta fluoretada, é um fator protetor capaz de minimizar os diferenciais entre classes ou níveis socioeconômicos. 4,5

A detecção dos diferenciais na condição de saúde bucal através do uso de categorias analíticas como o espaço social, definido por limites geográficos, administrativos ou indicadores sociais ainda é pouco comum nos estudos em saúde bucal.6,7

Resgatando o uso de variáveis socioeconômicas como preditoras da ocorrência de cárie dentária em populações no Brasil, a partir dos dados do levantamento em saúde bucal disponibilizados pelo Ministério da Saúde 8 para cada macro-região, Moysés ${ }^{9}$ tentou correlacionar o Índice de Desenvolvimento Humano (IDH) ao Índice Dentes Cariados, Perdidos e Obturados (CPO-D) obtido, considerando as desigualdades regionais. Ele encontrou uma correlação de $22 \%$ entre os dois indicadores, mostrando razoável poder explicativo do indicador, e o alto potencial dessa abordagem para detecção dos diferenciais entre os grupos. Afirma, também, que uma maior correlação pode não ter sido possível porque, mesmo dentro de cada macro-região, são significativas as diferenças entre os municípios, com uma grande heterogeneidade entre os estratos. ${ }^{9}$

Já Patussi10 realizou, no Distrito Federal, um estudo de associação entre cinco zonas, discriminadas pela renda média familiar e a prevalência de cárie dentária, examinando 1025 escolares de 12 anos de idade. A região A, com melhor condição socioeconômica, foi a que apresentou os índices mais favoráveis de cárie dentária, com um CPO-D = 1,8 (1,5-2,1) IC95\% e 43,7\% de crianças livres de cárie. Os piores resultados foram encontrados no estrato de renda intermediário (C), com um CPO-D = 3,5 (3,1$3,8)$ IC $95 \%$ e $16,2 \%$ de crianças livres de cárie, enquanto nas zonas $\mathrm{B}, \mathrm{D}$ e $\mathrm{E}$ os valores do CPO-D variaram entre 2,5 e 2,9, sem diferenças estatisticamente significativas, e com $24,3 \%$ a $30 \%$ de crianças livres de cárie.

Baldani et al.,11 correlacionaram, por meio da regressão linear simples, o CPO-D aos 12 anos de idade fornecidos por 357 municípios de estado do Paraná com índices globais como o IDH, Índice de Condições de Vida (ICV), Índice de Salubridade (IS), Índice de Desenvolvimento Infantil (IDI) além de outros indicadores relativos à renda, moradia e escolaridade. Os autores identificaram que 45,94\% dos municípios apresentavam alta prevalência de cárie e $28,01 \%$ classificavam-se como de prevalência moderada, com uma relação inversa entre severidade da doença e porte do município. Os níveis de cárie dentária e os indicadores globais de desenvolvimento apresentaram correlação negativa, indicando a tendência de municípios com melhores condições de vida apresentarem menores índices do agravo, e essa mesma correlação negativa se repetiu para as medidas de renda, indicadores de escolaridade, ligações à rede de abastecimento de água e aglomeração domiciliar.

Nessa perspectiva, este estudo visa analisar a prevalência e severidade da cárie dentária em Salvador, segundo distrito sanitário, de forma a identificar as iniqüidades na distribuição dessas doenças e contribuir para a programação em saúde bucal.

\section{Métodos}

O desenho epidemiológico foi o transversal. Para a análise do nível socioeconômico e caracterização do perfil epidemiológico e desigualdades da doença no município, se utilizaram-se as áreas definidas, geográfica e administrativamente pelos distritos sanitários (em número de 12), unidade operacional e gerencial utilizada pela Secretaria Municipal de Saúde para o planejamento e desenvolvimento das ações de saúde. 


\section{População de estudo}

A população de referência constituiu-se de escolares de $12(\mathrm{~N}=51.560)$ e $15 \operatorname{anos}(\mathrm{N}=52.420)$, de ambos os sexos, regularmente matriculados em escolas públicas e privadas de Salvador, Bahia, Brasil, ano de 2001.12 Esse número representava $97 \%$ das crianças aos 12 anos residentes no município e $95 \%$ aos 15 anos.

Essas idades foram selecionadas porque a World Health Organization (WHO) ${ }^{13}$ considera os 12 anos como idade de comparação e vigilância internacional das doenças bucais, e os 15 anos, como a idade na qual a prevalência de cárie é mais significativa, visto que os dentes permanentes estão expostos de três a nove anos no ambiente bucal.

Optou-se pela obtenção de uma amostra probabilística em múltiplo estágio, cujas unidades amostrais primárias representavam o cadastro de escolas públicas e particulares da cidade, fornecida pelo Ministério da Educação. Foram sorteados 72 estabelecimentos de ensino (10\% do total), pré-estratificados por Distrito Sanitário, obedecendo a proporcionalidade entre escolas públicas e privadas, de ensino fundamental e médio.

A fim de gerar medidas descritivas significativas por área geográfica e para o município de Salvador, para o cálculo da amostra foram usados os dados populacionais mais recentes disponíveis da média e variabilidade de cárie dentária da população aos 12 anos de idade (CPO-D $=1,53$; DP $=2,35$ ) para a cidade, 14 e as informações contidas no manual WHO, 13 que preconiza o mínimo de 25 crianças em regiões com baixa prevalência de cárie. Optou-se por dobrar esse número, com o objetivo de estabelecer segurança em relação a perdas e recusas de participação.

Foram considerados 12 estratos de análise, o número de distritos sanitários da cidade, e a unidade como número mínimo capaz de detectar-se a diferença entre os estratos. O nível de significância adotado foi de $5 \%$ e o poder do teste foi de $80 \%$. A distribuição $t$ de Student foi considerada a mais pertinente para o cálculo da amostra, conforme descrito:

$$
\mathrm{n} \geq \frac{2 \mathrm{~s}^{2}}{\delta^{2}}\left(\mathrm{t}_{\alpha, \mathrm{v}}+\mathrm{t}_{1-\beta, \mathrm{v}}\right)^{2}, \text { sendo: }
$$

$\mathrm{n}=$ amostra mínima requerida

$\mathrm{v}=$ variabilidade entre os grupos $=\mathrm{gn}_{\mathrm{o}}-\mathrm{g}$ (sendo $\mathrm{n}_{\mathrm{o}}$ $=50 \mathrm{e} \mathrm{g}=12)=588$

$\alpha=$ nível de significância $=5 \%$

$\beta=$ erro aceitável na hipótese alternativa $=20 \%$

$\delta=$ diferença mínima detectável na unidade do es- tudo $=1$

$\mathrm{s}=$ desvio padrão $=2,35$

$\mathrm{t}_{\alpha, \mathrm{v}}+1,96 \quad \mathrm{t}_{1-\beta, \mathrm{v}}=1,282$

Obteve-se uma amostra mínima de 117 adolescentes por idade e por distrito sanitário. De posse das listagens de alunos em cada escola foram selecionadas aleatoriamente as crianças em cada idade, com reposição única em casos de evasão escolar. Assim a amostra mínima total prevista foi de 2808 adolescentes, 1404 aos 12 anos e 1404 aos 15 anos de idade.

\section{Calibração da equipe}

O percentual de concordância geral foi de $98,16 \%$ e o kappa $=0,89$, sendo a pior concordância entre os examinadores de $85,30 \%$. Ambos os indicadores foram calculados a partir da média de concordância para cada dois examinadores. Observa-se que, se obteve uma concordância considerada satisfatória pela $\mathrm{WHO}^{11}$ para os levantamentos epidemiológicos em saúde bucal.

\section{Aspectos éticos}

O desenvolvimento deste estudo seguiu os requisitos da Resolução 196/96 do Conselho Nacional de Saúde, e as normas internas da Faculdade de Saúde Pública da USP, nos quais os adolescentes só foram examinados após a obtenção da autorização dos pais ou responsáveis dos alunos, através do termo de consentimento.

\section{Coleta de dados}

Os exames foram realizados no próprio ambiente escolar, durante o período letivo, obtendo-se as seguintes informações:

Dados gerais: nome, data de nascimento, tipo de financiamento da escola (pública, privada), série em curso, localização geográfica da escola, local de moradia nos primeiros cinco anos de vida, participação em outros programas com uso de flúor tópico e acesso à assistência odontológica no último ano e tipo de financiamento da mesma (se público, particular ou convênio).

Exame: foram inspecionadas as condições da coroa em relação à cárie, segundo os critérios estabelecidos pela WHO. 13

\section{Processo de análise}

Os dados foram digitados e armazenados num banco 
de dados. A análise foi realizada no Epi-info $6.04 \mathrm{e}$ no pacote estatístico STATA, versão 6.0®. Procurouse identificar as especificidades dos distritos sanitários e relação da prevalência e severidade das alterações estudadas utilizando a seguinte análise:

Distribuição da prevalência e severidade de cárie aos 12 anos segundo distritos sanitários;

Regressão logística não-condicional, com o modelo saturado composto das seguintes variáveis.

\section{Variáveis dependentes}

Prevalência de cárie dental (CPO-D $=0$ e CPO-D $>=$ 1) por distrito sanitário, por idade.

\section{Variável independente principal}

Distrito sanitário em que está localizada a escola ( 0 a 11): optou-se pelo uso dessa variável visto que o desenvolvimento de práticas de promoção de saúde bucal se efetivam, no município, em sua maior parte, no ambiente escolar.

\section{Covariáveis}

Sexo: masculino e feminino; etnia: negra; parda; branca e amarela, que foi excluída da análise por representar apenas um indivíduo em toda amostra, segundo critérios do Fundação Instituto Brasileira de Geografia e Estatística (IBGE) na amostragem por domicílio no ano 2000; participação em atividade de promoção de saúde/prevenção nos últimos dois anos com uso do flúor tópico: sim; não/não sabe informar; local de residência nos primeiros cinco anos de vida: Salvador; outros municípios e residiu somente parte do tempo em Salvador; acesso ao consultório odontológico no último ano: sim, não/não sabe informar; tipo de financiamento da assistência odontológica: público, particular; convênio/credenciamento, nunca teve acesso.

\section{Resultados}

Foram examinados 3318 adolescentes de escolas públicas e privadas de Salvador, e após a revisão e digitação das fichas, foram excluídas cinco por falta de preenchimento do campo "idade". Totalizou-se, assim 3313 àquelas utilizadas efetivamente para análise, sendo 1750 aos 12 anos e 1563 aos 15 anos (Tabela 1). O CPO-D aos 12 anos de idade foi de 1,44 e 2,66 aos 15 anos de idade, com a predominância do componente cariado e do obturado respectivamente. Quando se analisou a prevalência de cárie dentária, 49\% (46,66 51,34) IC95\% das crianças aos 12 anos encontravam-se livres de cárie, enquanto que aos 15 anos esta proporção correspondeu a $34,9 \%(32,53 \quad 37,27)$ IC95\%. (Tabelas 2 e 3 )

Quando se analisou a distribuição da cárie dentária por distrito sanitário, destacou-se as menores médias do CPO-D e a maior proporção de indivíduos livres de cárie nos distritos de Cajazeiras, Pau da Lima e Subúrbio Ferroviário aos 12 anos, com uma severidade classificada como muito baixa segundo os critérios da Organização Mundial da Saúde, de um CPO-D $<1,2$. Os outros distritos colocaram-se na categoria de prevalência baixa, isto é, um CPO-D entre 1,2 e 2,6. (Figura 1)

Aos 15 anos de idade, quando se analisa o CPO-D e seu respectivo intervalo de confiança, apenas Pau da Lima, Cajazeiras e o Subúrbio Ferroviário se mantiveram com menor experiência de cárie dental, sendo que os distritos Barra-Rio Vermelho, Itapagipe, Boca do Rio, Cabula-Beiru, Subúrbio Ferroviário e São Caetano-Valéria apresentaram-se em níveis intermediários. Já os distritos do Centro Histórico, Brotas, Itapoã e Liberdade foram identificados com maior experiência da doença. (Figuras 3 e 4).

$\mathrm{Na}$ regressão logística considerou-se a prevalência de cárie dentária, utilizando como referência o distrito sanitário de melhor condição de saúde bucal (Pau da Lima), detectou-se diferenças estatisticamente significantes entre os distritos, ajustadas pelas variáveis sexo e acesso ao dentista no último ano. Além dessas nenhuma das covariáveis propostas no modelo saturado foram importantes na associação principal. Destaca-se os Centro Histórico, Brotas, Itapoã, Subúrbio Ferroviário com cerca de 1,5 vezes mais ocorrência de cárie do que o referente; BarraRio Vermelho, Itapagipe e São Caetano-Valéria com duas vezes maior prevalência e a Liberdade, com a pior condição de saúde bucal. (Tabelas 4 e 5) 
Caracterização da população de estudo aos 12 e 15 anos de idade segundo tipo de escola, sexo, etnia, visita ao dentista no último ano, tipo de financiamento da mesma, participação em programas com uso do flúor e local de moradia nos primeiros cinco anos de vida. Salvador, Bahia, 2001.

\begin{tabular}{|c|c|c|c|c|}
\hline \multirow{2}{*}{ Variáveis } & \multicolumn{2}{|c|}{$12 \operatorname{anos}(n=1750)$} & \multicolumn{2}{|c|}{$15 \operatorname{anos}(n=1563)$} \\
\hline & $\mathrm{n}$ & $\%$ & $\mathrm{n}$ & $\%$ \\
\hline \multicolumn{5}{|l|}{ Tipo de Escola } \\
\hline Pública & 1315 & 75,1 & 1139 & 72,9 \\
\hline Particular & 435 & 24,9 & 424 & 27,1 \\
\hline \multicolumn{5}{|l|}{ Sexo } \\
\hline Masculino & 797 & 45,5 & 661 & 42,3 \\
\hline Feminino & 953 & 54,5 & 902 & 57,7 \\
\hline \multicolumn{5}{|l|}{ Etnia } \\
\hline Branco & 88 & 5,0 & 73 & 4,7 \\
\hline Negro & 414 & 23,7 & 345 & 22,1 \\
\hline Pardo & 1247 & 71,2 & 1145 & 73,2 \\
\hline Amarelo & 1 & 0,1 & - & - \\
\hline \multicolumn{5}{|c|}{ Visita ao dentista último ano } \\
\hline Sim & 902 & 51,5 & 741 & 47,4 \\
\hline Não/não sabe & 848 & 48,5 & 822 & 52,6 \\
\hline \multicolumn{5}{|l|}{ Financiamento } \\
\hline Público & 303 & 17,3 & 206 & 13,2 \\
\hline Particular & 279 & 15,9 & 268 & 17,1 \\
\hline Convênio & 250 & 14,3 & 242 & 15,5 \\
\hline Não sabe/não se aplica & 918 & 52,5 & 847 & 54,2 \\
\hline \multicolumn{5}{|c|}{ Participação programas coletivos últimos dois anos } \\
\hline Sim & 695 & 39,7 & 432 & 27,7 \\
\hline Não & 1055 & 60,3 & 1131 & 72,3 \\
\hline \multicolumn{5}{|c|}{ Local de moradia dos 0 aos 5 anos de idade } \\
\hline Salvador & 1481 & 84,7 & 1278 & 81,8 \\
\hline Outros & 269 & 15,3 & 285 & 18,2 \\
\hline
\end{tabular}

Tabela 2

Condição dentária: Índice de Dentes Cariados, Perdidos, Obturados (CPO-D) e intervalo de confiança a 95\% (IC95\%), Índice de Cárie Significante, percentagem de indivíduos livres de cárie e intervalo de confiança a $95 \%$ em escolares de 12 anos de idade segundo distrito sanitário. Salvador, Bahia, 2001.

\begin{tabular}{|c|c|c|c|c|c|}
\hline Distrito Sanitário & $\mathrm{n}$ & CPO-D & IC95\% & $\begin{array}{l}\text { \%livres } \\
\text { cárie }\end{array}$ & IC95\% \\
\hline Barra-Rio Vermelho & 202 & 1,44 & $1,20-1,68$ & 46,50 & $39,62-53,38$ \\
\hline Centro histórico & 134 & 1,60 & $1,26-1,94$ & 46,30 & $37,77-54,83$ \\
\hline Itapagipe & 150 & 1,51 & $1,22-1,80$ & 43,30 & $35,29-51,31$ \\
\hline Brotas & 141 & 1,48 & $1,15-1,81$ & 50,40 & $42,06-58,74$ \\
\hline Boca do Rio & 146 & 1,53 & $1,22-1,84$ & 48,60 & $40,41-56,79$ \\
\hline Itapoã & 127 & 1,82 & $1,47-2,17$ & 37,80 & $29,28-46,32$ \\
\hline Cajazeiras & 140 & 1,14 & $0,80-1,48$ & 60,70 & $52,53-68,87$ \\
\hline Cabula-Beiru & 144 & 1,40 & $1,11-1,69$ & 49,30 & $41,05-57,55$ \\
\hline Liberdade) & 112 & 1,91 & $1,48-2,34$ & 35,70 & $26,74-44,66$ \\
\hline Pau da Lima & 137 & 1,13 & $0,84-1,42$ & 63,50 & $55,36-71,64$ \\
\hline Subúrbio Ferroviário & 164 & 1,13 & $0,87-1,39$ & 55,50 & $47,89-63,11$ \\
\hline São Caetano-Valéria & 153 & 1,39 & $1,11-1,67$ & 47,70 & $39,79-55,61$ \\
\hline Total & 1.750 & 1,44 & $1,35-1,52$ & 49,00 & $46,66-51,34$ \\
\hline
\end{tabular}


Tabela 3

Condição dentária: Índice de Dentes Cariados, Perdidos, Obturados (CPO-D) e intervalo de confiança a 95\%, percentagem de indivíduos livres de cárie e intervalo de confiança a 95\% em escolares de 15 anos de idade segundo distrito sanitário em Salvador, Bahia, 2001.

\begin{tabular}{|c|c|c|c|c|c|}
\hline Distrito Sanitário & $\mathrm{n}$ & CPO-D & $\mathrm{IC} 95 \%$ & $\begin{array}{c}\text { \%livres } \\
\text { cárie }\end{array}$ & $\mathrm{IC} 95 \%$ \\
\hline Barra-Rio Vermelho & 174 & 2,56 & $2,18-2,94$ & 35,60 & $28,49-42,71$ \\
\hline Centro Histórico & 137 & 3,10 & $2,52-3,68$ & 27,00 & $19,49-34,51$ \\
\hline Itapagipe & 109 & 2,29 & $1,77-2,81$ & 37,60 & $28,41-46,79$ \\
\hline Brotas & 140 & 3,35 & $2,79-3,91$ & 22,10 & $15,16-29,04$ \\
\hline Boca do Rio & 111 & 2,27 & $1,72-2,82$ & 42,30 & $33,02-51,58$ \\
\hline Itapoã & 139 & 3,10 & $2,53-3,67$ & 26,60 & $19,18-34,02$ \\
\hline Cajazeiras & 148 & 1,82 & $1,47-2,17$ & 45,30 & $37,20-53,40$ \\
\hline Cabula-Beiru & 140 & 2,40 & $1,91-2,89$ & 32,10 & $24,29-39,91$ \\
\hline Liberdade & 117 & 2,87 & $2,35-3,39$ & 24,80 & $16,90-32,70$ \\
\hline Pau da Lima & 110 & 1,40 & $1,04-1,76$ & 51,80 & $42,37-61,23$ \\
\hline Subúrbio Ferroviário & 131 & 2,10 & $1,67-2,53$ & 37,40 & $29,03-45,77$ \\
\hline São Caetano-Valéria & 107 & 1,95 & $1,47-2,43$ & 40,20 & $30,82-49,58$ \\
\hline Total & 1563 & 2,46 & $2,52-2,80$ & 34,90 & $32,53-37,27$ \\
\hline
\end{tabular}

\section{Figura 1}

Índice de Dentes Cariados, Perdidos, Obturados (CPO-D) e intervalo de confiança a 95\% (segmentos verticais) em escolares de 12 anos de idade segundo distrito sanitário. Salvador, Bahia, 2001.

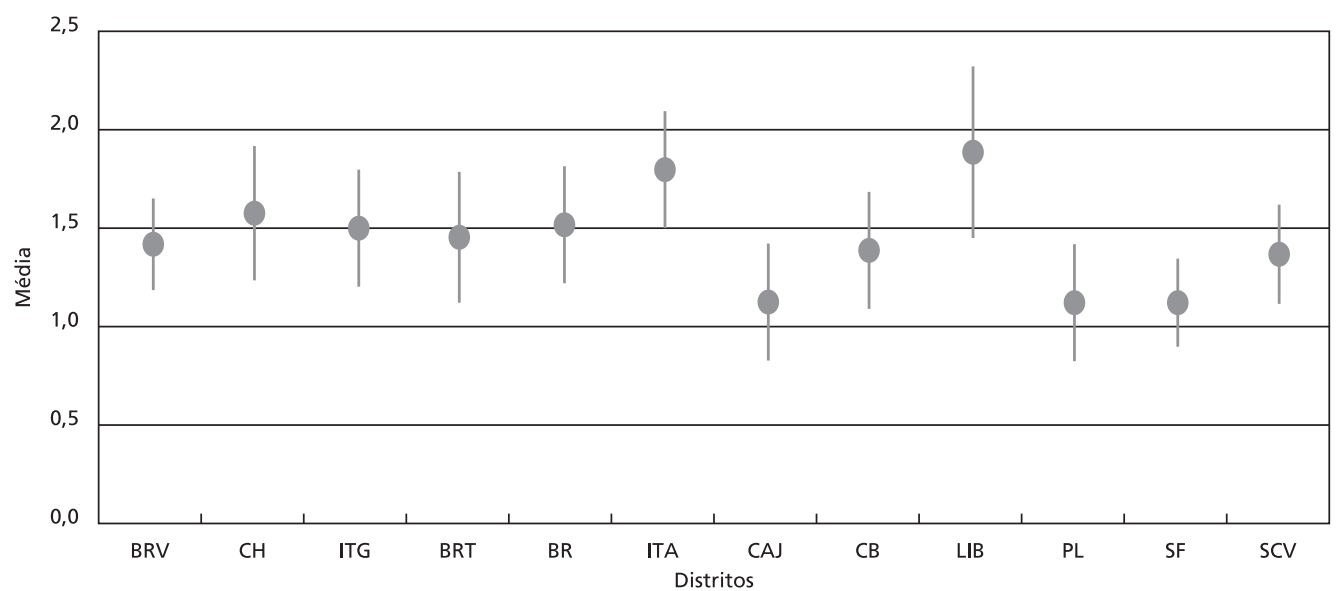

BRV = Barra-Rio Vermelho; $\mathrm{CH}=$ Centro Histórico; ITG = Itapagipe; BRT = Brotas; $\mathrm{BR}=$ Boca do Rio; ITA = Itapuã;

$C A J=$ Cajazeiras; $C B=$ Cabula-Beiru; $L I B=$ Liberdade; PL = Pau da Lima; SF = Subúrbio Ferroviário;

SCV = São Caetano-Valéria 
Índice de Dentes Cariados, Perdidos, Obturados (CPO-D) e intervalo de confiança a 95\% (segmentos verticais) em escolares de 12 anos de idade segundo distrito sanitário. Salvador, Bahia, 2001.

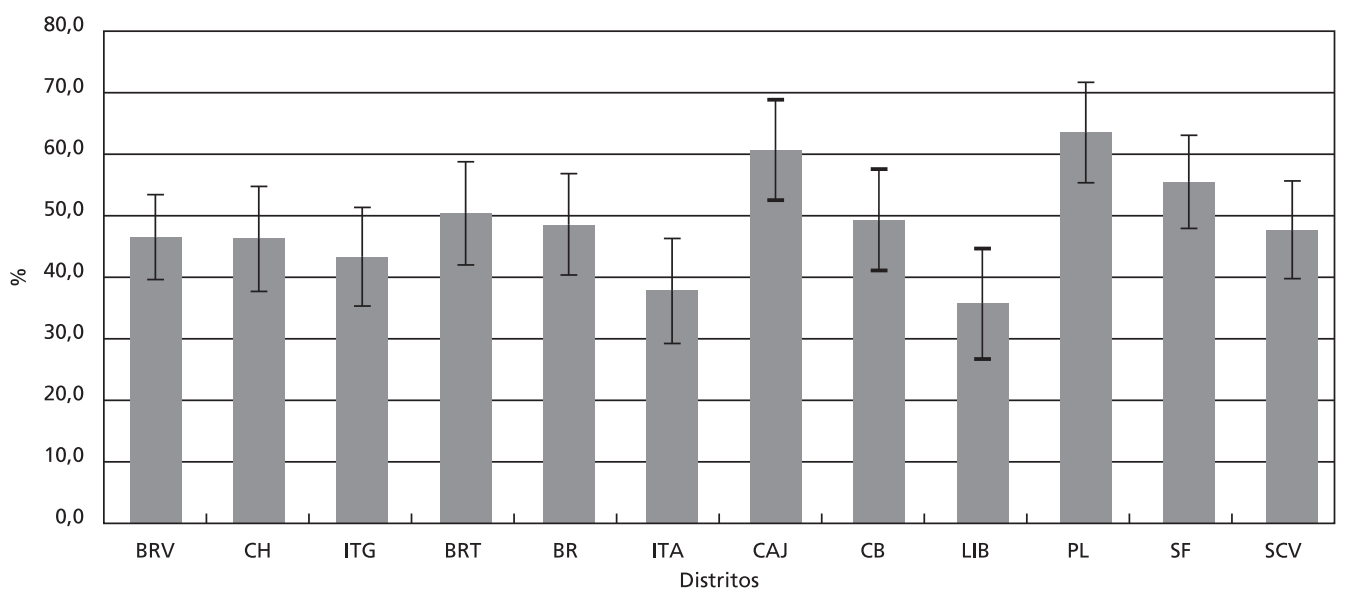

BRV = Barra-Rio Vermelho; $\mathrm{CH}=$ Centro Histórico; ITG = Itapagipe; $\mathrm{BRT}=$ Brotas; $\mathrm{BR}=$ Boca do Rio; ITA = Itapuã; $\mathrm{CAJ}=$ Cajazeiras; $C B=$ Cabula-Beiru; $\mathrm{LIB}=$ Liberdade; PL = Pau da Lima; SF = Subúrbio Ferroviário;

$\mathrm{SCV}=$ São Caetano-Valéria

\section{Figura 3}

Índice médio de Dentes Cariados, Perdidos, Obturados (CPO-D) e intervalo de confiança a $95 \%$ (segmentos verticais) em escolares de 15 anos de idade segundo distrito sanitário. Salvador, Bahia, 2001.

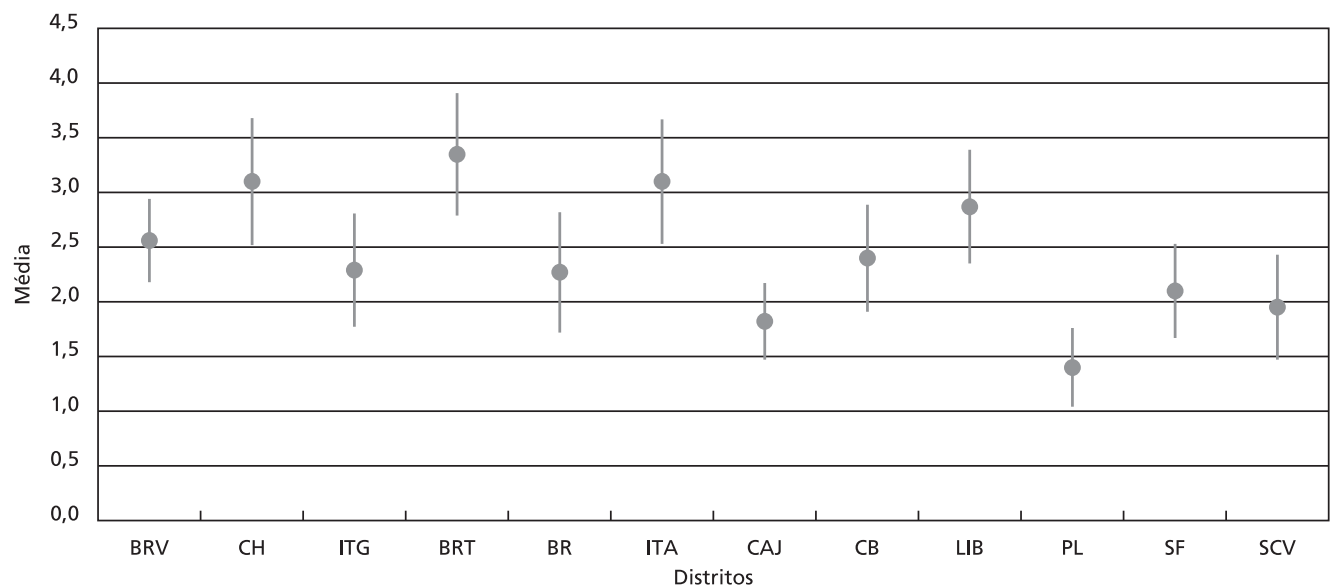

BRV = Barra-Rio Vermelho; $\mathrm{CH}=$ Centro Histórico; ITG = Itapagipe; BRT = Brotas; $\mathrm{BR}=$ Boca do Rio; ITA = Itapuã; $\mathrm{CAJ}=$ Cajazeiras; $\mathrm{CB}=$ Cabula-Beiru; $\mathrm{LIB}=$ Liberdade; PL = Pau da Lima; SF = Subúrbio Ferroviário;

SCV = São Caetano-Valéria 
Percentual de escolares livres de cárie e Intervalo de confiança 95\% (segmentos verticais) aos 15 anos de idade segundo distrito sanitário em Salvador Bahia, 2001.

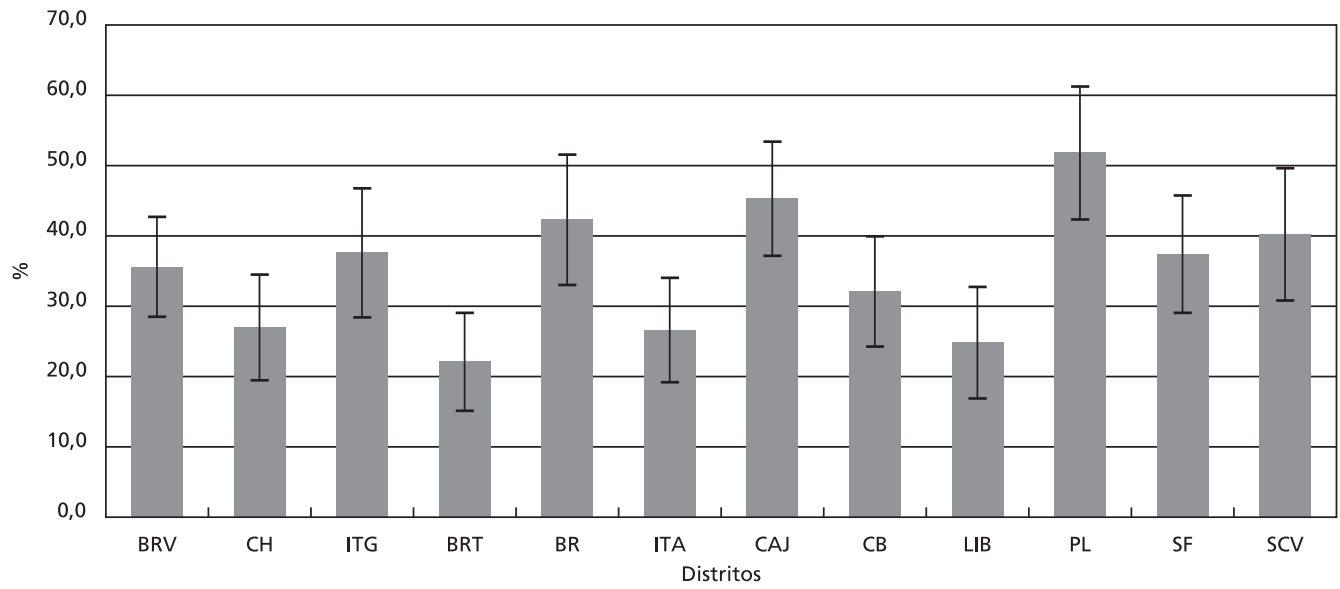

BRV = Barra-Rio Vermelho; $\mathrm{CH}=$ Centro Histórico; ITG = Itapagipe; BRT = Brotas; BR = Boca do Rio; ITA = Itapuã;

$\mathrm{CAJ}=$ Cajazeiras; $\mathrm{CB}=$ Cabula-Beiru; $\mathrm{LIB}=$ Liberdade; PL = Pau da Lima; SF = Subúrbio Ferroviário;

SCV = São Caetano-Valéria

Tabela 4

Modelo reduzido da regressão logística para a associação entre prevalência de cárie dentária (CPO-D=0) aos 12 anos 1 de idade segundo distrito sanitário ajustado por sexo e acesso ao dentista no último ano. Salvador, Bahia, 2001.

\begin{tabular}{|c|c|c|c|c|}
\hline Variáveis (Distritos) & OR Bruto & IC95\% & $\begin{array}{c}\text { OR } \\
\text { Ajustado }\end{array}$ & IC95\% \\
\hline Pau da Lima & 1,00 & - & 1,00 & - \\
\hline Barra-Rio Vermelho & 1,74 & $1,16-2,97$ & $1,93 *$ & $1,23-3,03$ \\
\hline Centro Histórico & 1,65 & $0,94-2,56$ & $1,72 *$ & $1,05-2,83$ \\
\hline Itapagipe & 2,22 & $1,37-3,62$ & $2,24 *$ & $1,39-3,64$ \\
\hline Brotas & 1,44 & $0,84-2,32$ & 1,65 & $1,01-2,68$ \\
\hline Boca do Rio & 1,43 & $0,87-2,33$ & 1,61 & $0,99-2,62$ \\
\hline Itapoã & 2,61 & $1,56-4,36$ & $2,69 *$ & $1,62-4,48$ \\
\hline Cajazeiras & 1,19 & $0,70-1,98$ & 1,22 & $0,75-2,00$ \\
\hline Cabula-Beiru & 1,47 & $0,87-2,46$ & 1,57 & $0,97-2,56$ \\
\hline Liberdade & 3,01 & $1,77-5,13$ & $3,02 *$ & $1,78-5,13$ \\
\hline Subúrbio Ferroviário & 1,42 & $0,91-2,06$ & 1,62 & $1,01-2,16$ \\
\hline São Caetano-Valéria & 1,88 & $1,17-3,04$ & $1,88^{*}$ & $1,17-3,04$ \\
\hline
\end{tabular}

12 anos $1 \mathrm{R} 2=0,14 ; p=0,00$; Máxima verossimilhança: $p=0,15$; *Diferenças estatísticas no nível de 5\%; IC95\% = Intervalo de confiança 95\%; OR = Odds ratio; CPO-D = Dentes Cariados, Perdidos e Obturados 
Modelo reduzido da regressão logística para a associação entre prevalência de cárie dentária (CPO-D=0) aos 15 anos 1 de idade segundo distrito sanitário ajustado por sexo e acesso ao dentista no último ano. Salvador, Bahia, 2001.

\begin{tabular}{|c|c|c|c|c|}
\hline Variáveis (Distritos) & OR Bruto & IC95\% & $\begin{array}{c}\text { OR } \\
\text { Ajustado }\end{array}$ & IC95\% \\
\hline Pau da Lima & 1,00 & - & 1,00 & -- \\
\hline Barra-Rio Vermelho & 1,40 & $1,00-3,16$ & 1,50 & $1,09-3,36$ \\
\hline Centro Histórico & 1,80 & $0,76-2,37$ & $1,92 *$ & $0,86-2,57$ \\
\hline Itapagipe & 1,18 & $0,93-6,89$ & 1,48 & $1,13-5,89$ \\
\hline Brotas & 3,1 & $0,36-1,96$ & $3,38 *$ & $0,56-1,70$ \\
\hline Boca do Rio & 0,97 & $1,27-3,81$ & 0,97 & $1,27-3,81$ \\
\hline Itapoã & 2,00 & $0,40-4,67$ & $2,20 *$ & $0,60-3,67$ \\
\hline Cajazeiras & 1,00 & $1,04-3,00$ & 1,00 & $1,04-3,00$ \\
\hline Cabula- Beiru & 1,50 & $1,21-4,27$ & $1,77 *$ & $1,41-4,47$ \\
\hline Liberdade & 2,04 & $0,52-2,23$ & 2,51 * & $0,90-2,60$ \\
\hline Subúrbio Ferroviário & 1,37 & $0,48-2,02$ & 1,53 & $0,73-2,23$ \\
\hline São Caetano-Valéria & 1,18 & $0,81-2,63$ & 1,28 & $0,91-2,48$ \\
\hline
\end{tabular}

15 anos $1 R^{2}=0,14 ; p=0,00 ;$ Máxima verossimilhança: $p=0,19$; Diferenças estatísticas no nível de 5\%; IC95\% = Intervalo de confiança 95\%; OR = Odds ratio; CPO-D = Dentes Cariados, Perdidos e Obturados

\section{Discussão}

Na tentativa de detectar as desiguais distribuições da cárie dentária no município, de forma a identificar grupos ou áreas epidemiologicamente susceptíveis, procedeu-se a análise das condições da cárie dentária, segundo distrito sanitário de localização da escola. Optou-se pela unidade escolar, e não o distrito de residência por ambos apresentarem uma boa concordância em quase todos os distritos e em função das estratégias de promoção e prevenção à saúde bucal serem desenvolvidas, prioritariamente, no ambiente escolar. 15

O distrito sanitário que apresentou melhor condição em relação à cárie dentária foi o de Pau da Lima, tanto aos 12 (CPO-D = 1,13; 63,5\% livres de cárie) como aos 15 anos de idade (CPO-D = 1,4; $51,8 \%$ livres de cárie). Esse distrito foi o primeiro a ser constituído no município, a partir de um convênio de cooperação técnica entre Brasil e Itália e vem, desde então, tentando desenvolver ações de vigilância à saúde, incluindo a saúde bucal, se constituindo também em campo de prática para o programa de residência multiprofissional em saúde da família do Instituto de Saúde Coletiva da Universidade Federal da Bahia, de 1998 a 2001.16 Por isso ele foi utilizado como referência para todos os pro- cedimentos analíticos desenvolvidos neste trabalho, considerando que maior severidade encontrada em outras áreas poderiam ser consideradas injustas ou evitáveis, isto é, passíveis de serem minimizadas.

Foi alta a proporção de indivíduos livres da doença aos 12 anos de idade, com piores condições nos distritos de Itapoã e Liberdade, que apresentaram também os CPO-D's mais elevados - 1,82 e 1,91 , respectivamente. A análise de regressão logística também sugere maior ocorrência de cárie nessas áreas, após o ajuste para as variáveis sexo e acesso ao dentista no último ano.

Dentre as possíveis hipóteses para essas diferenças, observa-se que o distrito de Itapoã, embora tenha apresentado um alto percentual de crianças que referiram participar de atividades de promoção da saúde bucal nos últimos dois anos, não possui nenhum registro dos mesmos na Secretaria Municipal de Saúde. Considera-se, também, que o mesmo possui uma rede assistencial pequena em relação ao porte do distrito, especialmente por ter sua área de abrangência ampliada ao atender municípios vizinhos, como Lauro de Freitas, Candeias e Camaçari. 16

A região da Liberdade tem uma das menores coberturas de programas com uso de fluoretos, aos 12 anos de idade, e também nenhum registro na Secretaria Municipal de Saúde do desenvolvimento dos mesmos, embora a sua rede assistencial seja con- 
siderada satisfatória. Embora não seja possível identificar através dos dados empíricos, observa-se que, naqueles distritos em que existe uma preocupação da gestão em saúde na tentativa de implantação de modelos de atenção à saúde de maior impacto epidemiológico, bem como redes sociais (Igreja, Organizações não governamentais, Escola) preocupados com o bem estar social, parecem também estar presentes melhores condições de saúde bucal.

Brotas, Centro Histórico, Itapoã e Liberdade são os distritos que, relativo ao grupo de 15 anos de idade se encontram com piores condições em relação à cárie dentária. Entretanto são menores as diferenças em relação a outras regiões de nível intermediário da doença, com melhores condições para os distritos da Boca do Rio, Pau da Lima e Cajazeiras.

As regiões de Pau da Lima e Boca do Rio apresentam as maiores coberturas dos programas de promoção da saúde bucal, tanto aos 12 como aos 15 anos, coerente com os dados apresentados pela Coordenação de Odontologia da Secretaria Municipal de Saúde, embora a mesma correlação não possa ser feita com o acesso ao dentista no último ano.

Assim, destaca-se a relevância de medidas preventivas como o acesso à água fluoretada e aos programas com uso de fluoretos extensivos a toda a população, bem como as estratégias de educação em saúde bucal no controle da cárie dentária, embora no presente estudo o impacto dessas ações não tenha sido mensurado individualmente. Ao analisar a assistência, foi observado que naqueles distritos onde o acesso ao dentista no último ano foi maior, elevouse também o componente restaurado do índice CPO$\mathrm{D}$, sendo necessárias investigações posteriores sobre o impacto epidemiológico que essa representa na condição de saúde bucal desse grupo, visto que outros trabalhos como os de Slade et al. ${ }^{4}$ e Gelbier, 5 Nadanovsky e Sheiham, 6 têm identificado a pouca participação da mesma para a melhoria das condições de saúde bucal, destacando inclusive o potencial iatrogênico que esta tem representado, quando associada a um modelo intervencionista e estritamente curativo-restaurador.

Como limites do estudo da cárie dentária observaram-se as dificuldades inerentes à utilização da variável distrito sanitário como representativa das desigualdades sociais no município. A heterogeneidade dos mesmos dificulta a interpretação dos dados em relação à probabilidade de adoecer. Entretanto, mesmo com o baixo poder analítico no teste de hipóteses, a comparação de indicadores regionais, permitiu a identificação de grupos mais susceptíveis e indicam a possibilidade de outros estudos etiológicos para detectar variáveis ou fatores associados aos agravos, bem como implementar medidas mais efetivas no controle da doença. Isto é, naqueles distritos em que foram identificadas piores condições em relação a cárie dentária, são necessárias intervenções mais expressivas no controle da doença, bem como a continuidade de ações populacionais, como a fluoretação das águas de abastecimento público e garantia de acesso a dentifrícios fluorados.

Além disso, inúmeras variáveis individuais e coletivas podem estar associadas ao agravo ou a área geográfica estudada, inclusive às discriminadoras do nível socioeconômico, como descrevem os trabalhos de Nadanovsky e Sheiham, 6 Jones et al.,7 Moysés, ${ }^{9}$ Patussi10 e Maltz e Silva, 17 e não foram abordadas no presente estudo.

Destaca-se também, como um aspecto muito relevante ao estudo, a não homogeneidade interna dentro de cada distrito sanitário, já que esta é uma área definida por critérios administrativos pelo setor saúde, e não sociais, convivendo de forma próxima áreas de níveis de renda extremos, ou de necessidades de saúde, visto que não são muito escassos os dados populacionais em saúde bucal disponíveis no município.

\section{Considerações finais}

Além da eqüidade em saúde, outros desafios colocam-se na construção de uma atenção à saúde bucal mais justa no município especialmente a garantia da universalidade do acesso e integralidade da atenção, ainda longe de serem alcançadas na estruturação dos sistemas locais de saúde, processo esse construído através de avanços e retrocessos na última década.

Dentre as possibilidades deste estudo, se ressalta a capacidade de produzir estimativas confiáveis nas idades de 12 e 15 anos para o município em relação à cárie dentária. Destaca-se a necessidade de aprofundamento deste trabalho na identificação de subgrupos de risco em cada distrito sanitário e de mapeamento de outras variáveis sociais, econômicas, culturais, comportamentais ou biológicas marcadoras dos agravos à saúde bucal. 


\section{Referências}

1. Costa MCN, Teixeira MGLC. A concepção do "espaço" na investigação epidemiológica. Cad Saúde Pública 1999; 15: 271-9.

2. Barmes DE. A global view of oral disease: today and tomorrow. Community Dent Oral Epidemiol 1999; 27: 2-7.

3. Dummer PMH. Factors influencing the caries experience of a group of children at ages of 11-12 and 15-16 years: results from an ongoing epidemiological survey. J Dent Res 1990; 18: 37-48.

4. Slade GD, Spencer AJ, Davies MJ, Stewart JF. Influence of exposure to fluoride water on socioeconomic inequalities in children's caries experience. Community Dent Oral Epidemiol 1996; 24: 89-100.

5. Gelbier S. National health service and social inequalities in dental health. Br Dent J 1998; 185: 28-9.

6. Nadanovski P, Sheiham A. Relative contribution of dental services to the change in caries levels of 12-year-old children in 18 industrialized countries in the 1970's and early 1980's. Community Dent Oral Epidemiol 1995; 23 : 331-9.

7. Jones CM, Woods K, Taylor GO. Social deprivation and tooth decay in Scottish schoolchildren. Health Bull 1997; 55: 11-5.

8. Ministério da Saúde. Resolução 196/96 do Conselho Nacional de Saúde sobre a regulamentação da pesquisa em seres humanos: 1997. Brasília (DF): O Ministério; 1997.

9. Moysés SJ. Desigualdade em saúde bucal e desenvolvimento humano: um ensaio em preto, branco e alguns tons de cinza. Rev Bras Odontol Saúde Coletiva 2000; 1: 7-17.
10. Pattusi MP. As desigualdades na distribuição da cárie dentária em escolares de 12 anos residentes em diferentes regiões socioeconômicas do Distrito Federal, Brasil, 1997. Rev Bras Odontol Saúde Coletiva 2000; 1: 19-28.

11. Baldani MH, Narvai PC, Antunes JLF. Cárie dentária e condições sócio-econômicas no Estado do Paraná, Brasil,1996. Cad Saúde Pública 2002; 18: 755-63.

12. Ministério da Educação e Cultural. Censo educacional 2000. Disponível em: <URL: htpps://www2.inep.gov.br>. [2001 jan 4].

13. WHO (World Health Organization). Oral health surveys: basic methods. Geneva: The Organization; 1997.

14. Ministério da Saúde. CPO-D médio nas capitais brasileiras, 1996. Disponível em: <URL: http:// www. saude.gov.br/programas/bucal>. [1999 out 20].

15. Cabral MBBS, Cangussu MCT, Liesenfeld MH, Vianna MIP. A construção democrática de um novo modelo de atenção à saúde bucal. Rev Bras Odontol Saúde Coletiva 2000: 55-62. [Edição especial].

16. Salvador. Secretaria de Saúde. Plano municipal de Saúde: 1994-1997. Salvador: A Secretaria; 1994.

17. Maltz M, Silva BB. Relação entre cárie, gengivite e fluorose e nível socioeconômico em escolares. Rev Saúde Pública 2001; 35: 170-6.

Recebido em 12 de dezembro de 2003

Versão final apresentada em 3 de maio de 2004

Aprovado em 27 de junho de 2004 